\title{
Acute Suppurative Thyroiditis Secondary to Escherichia coli Infection
}

\author{
Nida Siddiqui ${ }^{1}$, Nikola Deletic ${ }^{1}$, Frederick Raal ${ }^{1,2}$, Farzahna Mohamed $^{1,2}$ \\ ${ }^{1}$ Department of Internal Medicine, Faculty of Health Sciences, University of the Witwatersrand, Johannesburg, South Africa \\ ${ }^{2}$ Division of Endocrinology and Metabolism, Faculty of Health Sciences, University of the Witwatersrand, Johannesburg, South Africa
}

Received: $17 / 10 / 2021$

Accepted: $24 / 10 / 2021$

Published: 19/11/2021

How to cite this article: Siddiqui N, Deletic N, Raal F, Mohamed F. Acute suppurative thyroiditis secondary to Escherichia coli infection. EJCRIM 2021;8: doi:10.12890/2021_003009.

Conflicts of Interests: The authors declare there are no competing interests.

This article is licensed under a Commons Attribution Non-Commercial 4.0 License

\section{ABSTRACT}

Infections of the thyroid gland are rare. Its innate resistance to infections can be attributed to its unique anatomical features and rich blood supply. High clinical suspicion is required as a delay in diagnosis can lead to significant morbidity and mortality. Major pathogens include the Gram-positive Staphylococcus aureus and Streptococcus species; however, Gram-negative organisms have been found especially in immunocompromised hosts. We present a rare case of acute suppurative thyroiditis (AST) secondary to Escherichia coli (E. coli) infection in a woman known to be infected with human immunodeficiency virus (HIV).

\section{LEARNING POINTS}

- Thyroid abscesses are rare and can be confused with more common pathologies involving the neck such as a goitre, adenoma, intracystic haemorrhage, pharyngeal abscess and subacute thyroiditis.

- A high index of suspicion for a thyroid abscess is required for patients who present with an anterior neck swelling to avoid a late diagnosis, which is associated with significant morbidity and mortality.

- Acute suppurative thyroiditis is more commonly caused by Gram-positive organisms. Gram-negative organisms such as E. coli remain a rare cause. However, if a thyroid abscess is suspected clinically, broad-spectrum antibiotics can be lifesaving before definite culture and sensitivity results are available.

\section{KEYWORDS}

Thyroid abscess, thyroid, Escherichia coli, acute suppurative thyroiditis, abscess

\section{CASE DESCRIPTION}

A 51-year-old virally suppressed, HIV-infected woman with a CD4 count of 129 cells $/ \mathrm{mm}^{3}$ presented with a 3-week history of dysphagia, dyspnoea and progressive neck swelling which was tender and accompanied by hoarseness and diaphoresis. She denied any history of thyroid disease, surgery or trauma to the neck. Her past medical history was significant for hypertension and pulmonary tuberculosis for which she completed treatment. Her medications included amlodipine and tenofovir/lamivudine/dolutegravir.

On examination she had blood pressure of $128 / 68 \mathrm{mmHg}$ and tachycardia of $118 \mathrm{bpm}$. She ran a low-grade fever of $37.9^{\circ} \mathrm{C}$ and maintained $89 \%$ saturation on room air which improved with supplemental oxygen. The left lobe of the thyroid gland was enlarged, tender, firm to palpation and associated with bilateral cervical lymphadenopathy. There were no clinical signs of retrosternal extension of the thyroid gland. 
Methods and Procedures

Laboratory investigations were ordered which revealed elevated septic markers with a predominant neutrophilia and a negative blood culture. An elevated thyroxine level and decreased thyroid stimulating hormone was also noted (Table 1). A diagnosis of acute suppurative thyroiditis (AST), pharyngeal abscess and subacute thyroiditis was considered, and the patient commenced empiric amoxicillin/clavulanate which was later escalated to piperacillin/tazobactam covering an extended spectrum of Gram-negative bacteria including Pseudomonas aeruginosa, as well as Gram-positive and anaerobic bacteria.

Radiological investigations included a CXR for her dyspnoea which revealed tracheal deviation to the right (Fig. 1). A barium swallow was ordered to investigate the dysphagia and showed anterior deviation of the oesophagus (Fig. 2). The diagnosis was eventually confirmed by a contrasted computed tomography (CT) scan of the neck and chest which confirmed the presence of a thyroid abscess (Fig. 3).

\begin{tabular}{|c|c|c|c|c|}
\hline Investigation & Reference range & Presentation & 3 days post-aspiration & 8-month follow-up visit \\
\hline WCC $\left(\times 10^{9} / \mathrm{l}\right)$ & $3.90-12.60$ & 19.14 & 9.79 & 3.77 \\
\hline Neutrophils $\left(\times 10^{9} / \mathrm{l}\right)$ & $1.60-8.30$ & 18.57 & & 1.80 \\
\hline $\mathrm{CRP}(\mathrm{mg} / \mathrm{l})$ & $<10$ & 264 & 82 & $<10$ \\
\hline РCT $(\mu \mathrm{g} / \mathrm{l})$ & $<2.0$ & 7.61 & 0.29 & Not done \\
\hline $\mathrm{ESR}(\mathrm{mm} / \mathrm{hr})$ & $0-10$ & 85 & & 12 \\
\hline $\mathrm{TSH}(\mathrm{mlU} / \mathrm{l})$ & $0.27-4.20$ & 0.17 & & 1.35 \\
\hline Free T4 (pmol/l) & $12.0-22.0$ & 28.6 & & 14.5 \\
\hline Blood culture & & Positive (CNS)* & Negative & Not done \\
\hline
\end{tabular}

Table 1. Laboratory investigations on admission, following aspiration and on follow-up

WCC: White cell count; CRP: C-reactive protein; PCT: procalcitonin; ESR: erythrocyte sedimentation rate; TSH: thyroid stimulating hormone; T4: thyroxine; CNS: coagulase-negative Staphylococcus; * suspected contamination.

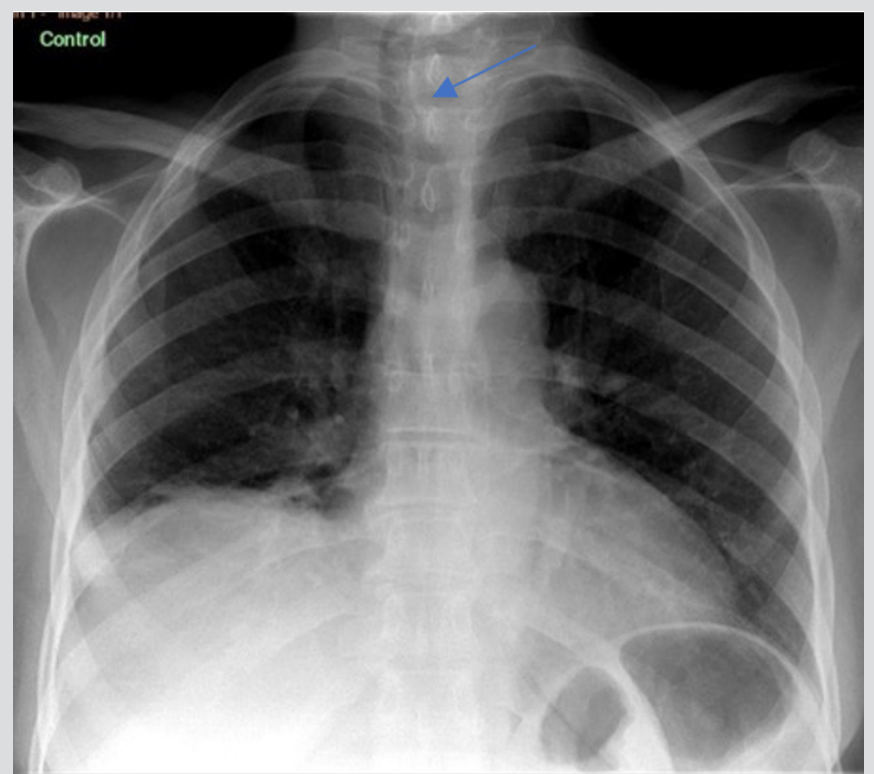

Figure 1. Chest $x$-ray showing tracheal deviation to the right (arrow)

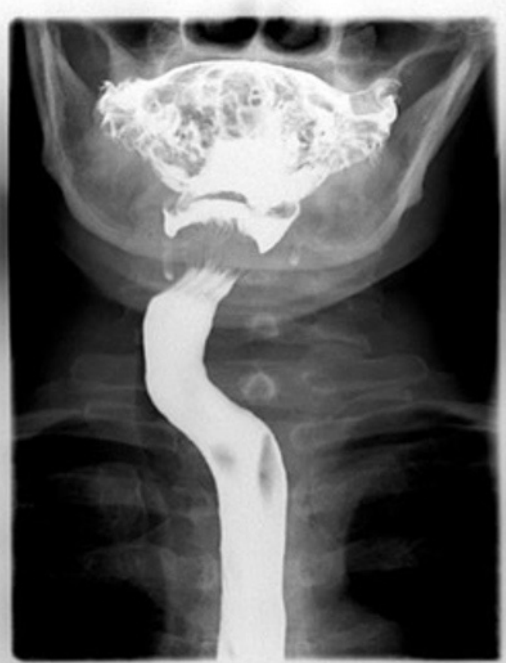

Figure 2. Barium swallow demonstrating anterior deviation of the oesophagus 
Ultrasound-guided fine needle aspiration (FNA) aspirated 22 millilitres of purulent fluid (Fig. 4) and microscopy, culture and sensitivity (MC\&S) grew Escherichia coli (Fig. 5) which was treated with piperacillin/tazobactam as per sensitivity. Repeat ultrasound showed minimal fluid not amenable to aspiration. Antibiotic therapy was continued until complete clinical resolution and an improvement in septic markers was seen. Urine MC\&S was not performed and thus the source of infection remains unknown. We postulate that the cause could be a partially treated urinary tract infection caused by E. coli. Her follow-up visit yielded an unremarkable examination with normal thyroid function tests. A repeat ultrasound showed no residual collections in the left thyroid lobe.

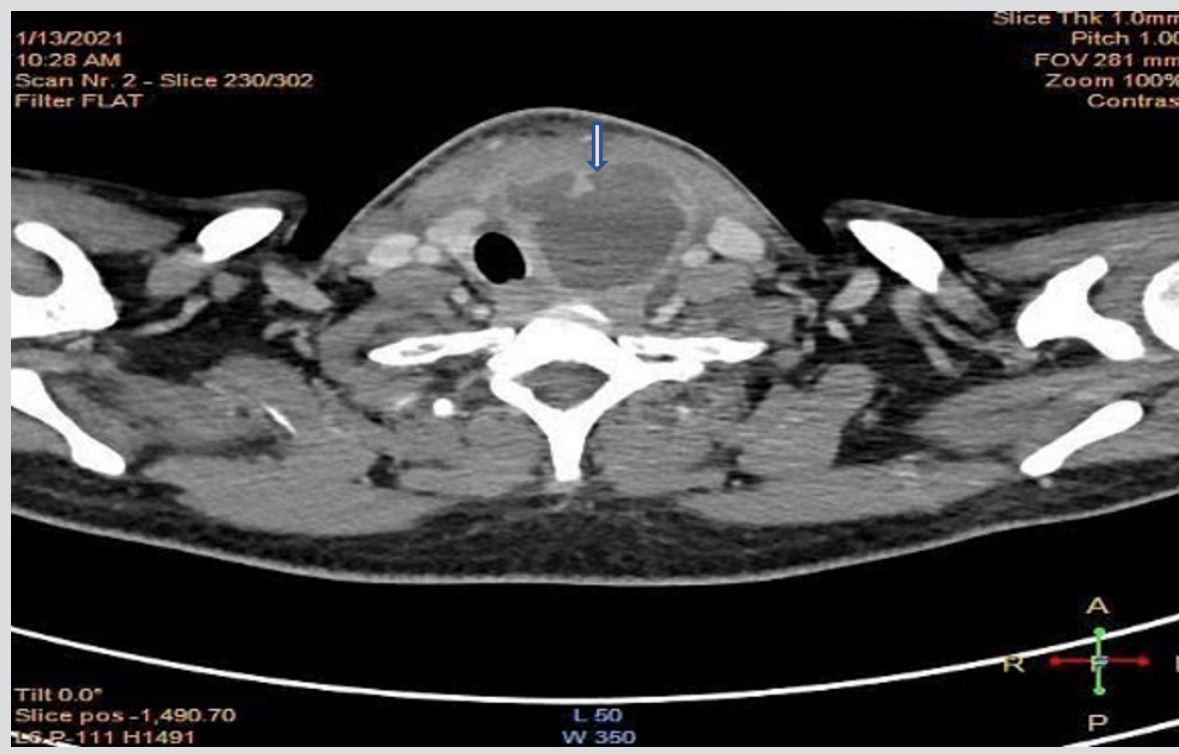

Figure 3. CT scan of the neck showing an enlarged, fluid-attenuated, necrotic mass (arrow) with minimal septation inseparable from the left thyroid lobe measuring $4.5 \times 5.1 \times 4.7 \mathrm{~cm}(\mathrm{AP} \times \mathrm{TV} \times \mathrm{CC})$. There was evidence of mass effect with deviation of the thyroid, larynx and trachea to the right, without compromising the airway. No underlying anatomic structural defect was found

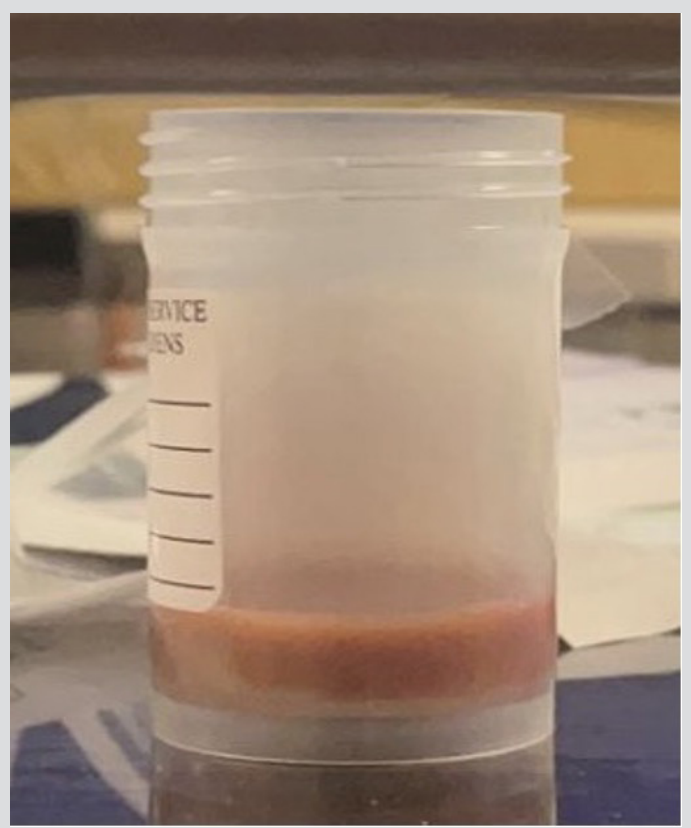

Figure 4. A sample of the red-brown coloured fluid aspirated from the abscess

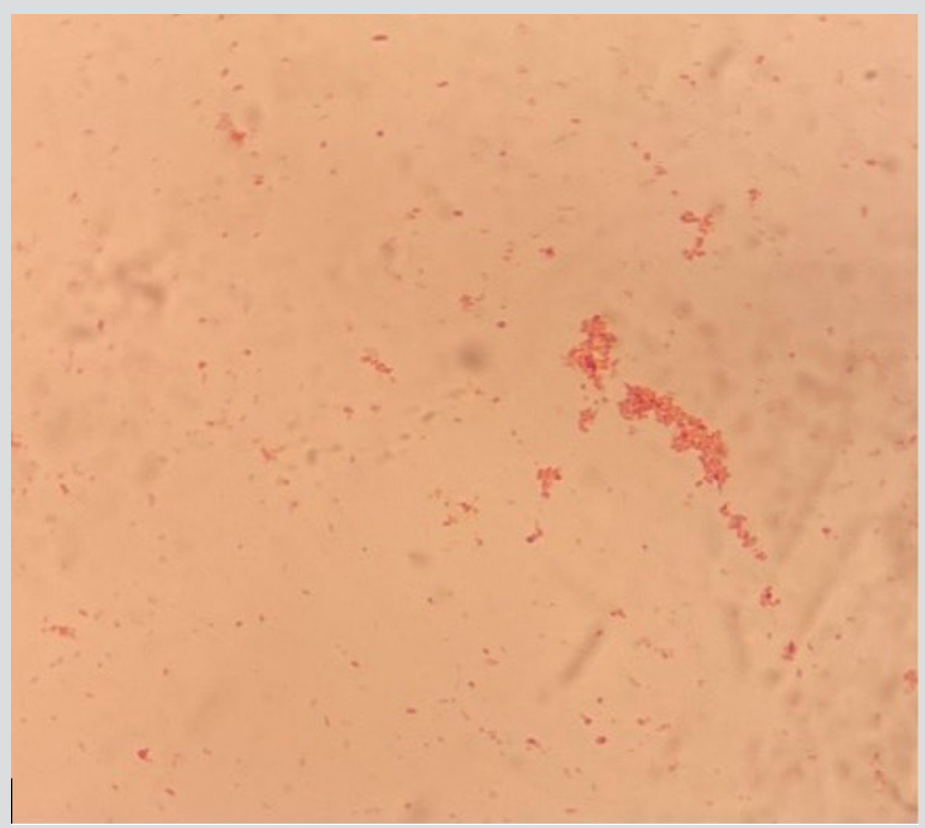

Figure 5. Gram stain of Escherichia coli

\section{DISCUSSION}

Thyroiditis is an inflammation of the thyroid gland and may have a variety of aetiologies ${ }^{[1]}$.

Factors such as capsular encasement, a rich vascular supply, good lymphatic drainage and the presence of iodine and hydrogen peroxide make the gland resistant to infections ${ }^{[1,2]}$. AST is a relatively uncommon form of thyroiditis with an incidence of $0.1-0.7 \%$ of all thyroid disorders ${ }^{[1,2]}$. 
It is usually caused by haematogenous spread or direct inoculation of pathogens, lymphatic spread from distant sites, direct trauma from a foreign body or contamination from an unsterile FNA ${ }^{[1,2]}$. Organisms typically found are Staphylococcus aureus and Streptococcus species, Mycobacterium tuberculosis, Klebsiella pneumoniae, Bacteroides spp., Pneumocystis jirovecii, Nocardia spp. and Candida. E. coli is a rare cause of $\mathrm{AST}^{[1-3]}$.

AST complicated by thyroid abscess is extremely rare and can be associated with life-threatening complications if left untreated. Thyroid abscess represents $<1 \%$ of surgically treated thyroid gland pathology ${ }^{[3,4]}$. It commonly occurs in immunocompromised patients, for example, those with HIV, on immunosuppressive therapy, with pre-existing thyroid or metabolic diseases or with congenital abnormalities such as pyriform sinus fistula ${ }^{[2,3]}$.

Patients may present acutely or may take a more chronic course depending on the underlying organism ${ }^{[5]}$. In a retrospective review, most patients with bacterial infection were found to be euthyroid whereas mycobacterial and fungal infections were found to be associated with hyper- and hypothyroid status, respectively ${ }^{[1]}$.

Ultrasound is the diagnostic modality of choice and provides a therapeutic benefit by facilitating drainage ${ }^{[1,3]}$. A CT scan or magnetic resonance imaging are considered if ultrasound is inconclusive or if spread to adjacent structures is clinically suspected ${ }^{[3]}$. Traditionally, AST with an abscess has been treated with partial/total thyroidectomy or surgical drainage combined with antibiotic therapy ${ }^{[1,3]}$. Surgical drainage is also indicated for persistent or recurrent abscesses.

Potential complications of a thyroid abscess include thyroid storm, internal jugular vein thrombosis, airway obstruction, tracheal or oesophageal perforation, necrotizing mediastinitis, sepsis and death ${ }^{[1,5]}$. Timely intervention is required to prevent potentially fatal consequences $^{[3]}$.

With effective antibiotic therapy and surgical drainage, patients typically have an excellent prognosis ${ }^{[1]}$. The outcome from AST has ranged from complete resolution to $12 \%$ or higher mortality in the absence of immediate intervention ${ }^{[3]}$. Exact morbidity and mortality is unknown due to its low incidence and paucity of retrospective studies, and more studies are needed to report on this aspect ${ }^{[3]}$.

\section{REFERENCES}

1. Singh G, Jaiswal R, Gulati N, Campbell Granieri E. A case of idiopathic thyroid abscess caused by Escherichia coli. J Community Hosp Intern Med Perspect 2019;9(2):159-161.

2. Sen S, Ramakant P, Paul MJ, Jennifer A. Acute suppurative thyroiditis secondary to urinary tract infection by E. coli: a rare clinical scenario. BMJ Case Rep 2016;2016:bcr2015213231.

3. Paes JE, Burman KD, Cohen J, Franklyn J, McHenry CR, Shoham S, et al. Acute bacterial suppurative thyroiditis: a clinical review and expert opinion. Thyroid 2010;20(3):247255.

4. Ghaemi N, Sayedi J, Bagheri S. Acute suppurative thyroiditis with thyroid abscess: a case report and review of the literature. Iran J Otorhinolaryngol 2014;26(74):51-55.

5. Brown J, Nguyen HH, Cohen SH. A pain in the neck: thyroid abscess. Am J Med 2014;127(3):e5-e 